\title{
Variants in TLR6 Gene Influence Susceptibility to Tuberculosis in the Chinese Population
}

\author{
Chao Ding \\ Xi'an Chest Hospital \\ Lin Wei \\ Xi'an Chest Hospital \\ Qian Li \\ Xi'an Chest Hospital \\ Yugang Liu \\ Xi'an Chest Hospital \\ Liming Duan \\ Xi'an Chest Hospital \\ Zhenning Gu \\ Xi'an Chest Hospital
}

Chaolei Guo ( $\sim$ professors12@163.com )

Xi'an Chest Hospital

\section{Research article}

Keywords: Tuberculosis, TLR6, Variant, Polymorphism, Genetic models

Posted Date: May 15th, 2020

DOl: https://doi.org/10.21203/rs.3.rs-28365/v1

License: (c) (i) This work is licensed under a Creative Commons Attribution 4.0 International License.

Read Full License 


\section{Abstract}

BACKGROUND Tuberculosis (TB) is a common chronic infectious disease caused by Mycobacteria tuberculosis. Several researches revealed that TLR6 play a crucial roles in the risk of TB.

METHODS The association between TLR6 polymorphism and TB risk were evaluated by a case-control study. Total of 510 TB cases and 508 unrelated healthy controls were recruited. Subsequently, the Agena MassARRAY platform was conducted to genotype, and odds ratios (ORs) and 95\% confidence intervals (Cls) were assessed the relationship between TLR6 variants and TB risk.

RESULTS We found that rs6531668 of TLR6 gene was significantly association with TB risk. Stratified analysis confirmed that rs6531668 was significant increased risk of TB in age $>41$ years old. Interestingly, the G/C - C/C genotype of rs 12642845 was significantly interacted with decreased risk of TB in age > 41 years. In males, rs 3775073 G/A genotype and rs 12642845 G/C genotype were correlated with decreased the risk of TB. Besides, we also observed that the CTA and TCG haplotypes were decreased TB risk.

CONCLUSIONS Our results shown that TLR6 gene variants were related with TB risk among the Chinese population.

\section{Instruction}

Tuberculosis (TB) is a common chronic pulmonary contagious human disease, and remains a highly mortality around the word. It was reported that TB causes a serious public health issue, multiple socioeconomic burden in our world, especially in Asia [1]. In 2017, according to the WHO calculated, approximately 10.0 million people suffered from TB and 1.3 million people died (Global tuberculosis report). It is estimated that approximately 5 to $15 \%$ of the infected individuals develop clinical disease during their lifetimes [2]. Various factors affect TB infection, including environment, genetic, immunosuppressive treatment, lifestyle and aging [3-6]. Previous twin studies, genome-wide association studies (GWAS) indicated that host heredity factors was considered to play an crucial role in the sensibility to TB [7]. Furthermore, human and mouse experiments have confirmed various potential TB risk loci, such as genes involved in toll-like receptor (TLR) signaling [8].

Toll-like receptors (TLRs) family are consist of 10 proteins that play an important role in our innate immune system. In human, these receptors are primarily identified pathogen-associated molecular patterns (PAMPs) and activate signaling cascades that caused the human innate immune response, cytokines and formation of the adaptive immune response $[9,10]$. Human genetic studies revealed that TLR pathway genes variants such as TLR1, TLR2, TLR4 and TLR9 have been confirmed with TB risk in different populations [11-13]. However, TLR6, a member of TLRs family. Less is known about the correlation between TLR6 polymorphisms and the risk of TB, particularly in Chinese Han population [14]. 
Here, we performed a case-control study to investigate six SNPs of TLR6 gene, including rs 3775073 , rs3796508, rs6531668, rs12644563, rs12640631, rs12642845. We verified the interaction of these SNPs with TB sensibility in a Chinese Han population from northwest China and our study are supposed to provide more evidence that can help elucidate TB pathogenesis among these individuals.

\section{Materials And Methods}

\section{Subjects}

The TB patients $(n=510)$ were recruited from Xi'an Chest Hospital, Shaanxi Province. According to positive sputum smear and some clinical signs, all of them were diagnosed by specialized doctors. TB patients including chronic inflammatory, HIV infection, primary immunodeficiency and other host immunity diseases were excluded. The healthy controls $(n=508)$ were matched to the TB patients by sex and age. In addition, we excluded healthy controls, who have TB history, autoimmune diseases and other chronic infections.

\section{Genotyping}

The blood samples $(5 \mathrm{~mL})$ were collected from each subject into a glass tube containing ethylenediaminetetraacetic acid (EDTA) for molecular analysis. Genomic DNA was extracted from whole blood samples by GoldMag whole blood genomic DNA kit (GoldMag, China). According to the specification, DNA purity was evaluated using NanoDrop 2000 platform (Thermo Fisher Scientific, USA). Base on the minor allele frequency $>0.05$, six candidate SNPs of $T L R 6$ gene were choose from the 1000 Genomes Project data (http://www.internationalgenome.org/). The SNPs primers were designed by Agena Bioscience Assay Design Suite V2.0 software. Furthermore, the MassARRAY iPLEX platform (Agena, USA) was conducted to genotype SNPs. All data was analyzed using Agena Bioscience TYPER version 4.0 software as previously our described [15].

\section{Statistical analysis}

SPSS 20.0 software was performed statistical analysis. Hardy-Weinberg equilibrium (HWE) was evaluated by $\chi^{2}$ test in healthy controls. We also compared allele and genotype frequencies using the $\chi^{2}$ test among all subjects. The association between the TLR6 polymorphism and the risk of TB was evaluated through odds ratios (ORs) and 95\% confidence intervals (Cls) adjustment gender and age. Linkage disequilibrium (LD) between SNPs were performed using Haploview 4.2 software. For all statistical tests, $p<0.05$ was considered statistically significant.

\section{Results}


The characteristics of the subjects were listed in Table 1. A total of 510 TB patients including 318 males and 192 females and 508 unrelated healthy controls involving 316 males and 192 females were employed. All participants were of Han ethnicity, and lived in or around the Xi'an city. The mean ages of TB cases and healthy controls were $41.90 \pm 14.83$ years old and $41.14 \pm 18.42$ years old, respectively. No significant differences between all individuals in age or gender proportion were observed.

Table 1

Demographic distribution of TB patients and Healthy controls

\begin{tabular}{|c|c|c|c|}
\hline & Cases $(n=510)$ No. $(\%)$ & Controls $(n=508)$ No. $(\%)$ & $\mathbf{p}$ \\
\hline Age, year $($ mean \pm SD $)$ & $41.90 \pm 14.83$ & $41.14 \pm 18.42$ & 0.469 \\
\hline Gender & & & 0.961 \\
\hline Male & $318(62.35)$ & $316(62.20)$ & \\
\hline Female & $192(37.65)$ & $192(37.80)$ & \\
\hline
\end{tabular}

In our study, the detail information of these SNPs (the position, minor allele frequency (MAF) and HWE $p$ value) were summarized in Table 2. We found that rs6531668 of TLR6 gene was association with TB risk $(\mathrm{OR}=1.28,95 \% \mathrm{Cl}=1.04-1.57, p=0.023)$. Analysis of the genotype frequencies of $T L R 6$ in different genetic models, our results suggested that rs6531668 was correlated with increased the risk of TB in dominant model $(\mathrm{OR}=1.30,95 \% \mathrm{Cl}=1.01-1.67, p=0.046)$ and $\log$-additive model $(\mathrm{OR}=1.26,95 \% \mathrm{Cl}=$ $1.03-1.55, p=0.026)$. However, no significant association was identified between rs $3775073, \mathrm{rs} 3796508$, rs12644563, rs12640631, rs12642845 and TB risk.

In stratified analysis by age, the results confirmed that rs 6531668 was increased TB risk in age $>41$ years under the co-dominant model $(\mathrm{OR}=1.75,95 \% \mathrm{Cl}=1.14-2.68, p=0.010)$, the dominant model $(\mathrm{OR}=1.77$, $95 \% \mathrm{Cl}=1.19-2.62, p=0.005)$ and the log-additive model $(\mathrm{OR}=1.51,95 \% \mathrm{Cl}=1.11-2.06, p=0.005)$. Interestingly, we observed that the $\mathrm{G} / \mathrm{C}-\mathrm{C} / \mathrm{C}$ genotype of rs12642845 was significantly interacted with decreased TB risk in age $>41$ years old under the dominant model $(\mathrm{OR}=0.67,95 \% \mathrm{Cl}=0.46-0.99, p=$ 0.042 ) (Table 4). When gender stratification was analyzed, the results confirmed that rs $3775073 \mathrm{G} / \mathrm{A}$ genotype and $\mathrm{rs} 12642845 \mathrm{G} / \mathrm{C}$ genotype were decreased TB risk in males $(\mathrm{OR}=0.70,95 \% \mathrm{Cl}=0.50-0.98$, $p=0.036 ; \mathrm{OR}=0.71,95 \% \mathrm{Cl}=0.51-0.99, p=0.042$, respectively). However, no association of rs3775073 and rs12642845 and TB risk were observed in females. The results were shown in Table 5.

We further performed LD and haplotype analyses of all SNPs in our study. Strong LD analysis displayed the existence of two blocks in TLR6 SNPs (Fig. 1). LD was found between rs3775073 and rs6531688, among rs12640631 and rs12642845. Besides, we observed that the CTA and TCG haplotypes were associated with reduced risk of TB $(\mathrm{OR}=0.74,95 \% \mathrm{Cl}=0.55-0.99, p=0.043 ; \mathrm{OR}=0.47,95 \% \mathrm{Cl}=0.28-$ $0.79, p=0.004$, respectively) (Table 6).

Moreover, we used Regulome DB and Haploreg v4.1 to determine the potential functional effect of these SNPs. The Regulome DB suggested that rs6531688 and rs3775073 were the binding site for the 
transcription factor. The Regulome DB score was $1 \mathrm{f}$ and $2 \mathrm{~b}$. No data were shown the prediction functional of rs3796508 and rs12640631 (Supplementary Table 1).

Table 2

Basic characteristics and allele frequencies among TLR6 SNPs

\begin{tabular}{|llllllll|}
\hline SNP & Chr & Allele & MAF & \multicolumn{5}{c|}{ HWE p-Value } & OR $(95 \% \mathrm{Cl})$ & $\mathrm{p}^{\mathrm{a}}$ \\
\cline { 5 - 7 } & & & Case & Control & & \\
\hline rs3775073 & 4 & A/G & 0.31 & 0.33 & 0.163 & $0.89(0.74-1.07)$ & 0.205 \\
\hline rs3796508 & 4 & A/G & 0.04 & 0.05 & 0.639 & $0.72(0.47-1.10)$ & 0.130 \\
\hline rs6531668 & 4 & A/G & 0.25 & 0.20 & 0.276 & $1.28(1.04-1.57)$ & $0.023^{*}$ \\
\hline rs12644563 & 4 & C/T & 0.04 & 0.05 & 0.148 & $0.72(0.47-1.10)$ & 0.130 \\
\hline rs12640631 & 4 & C/T & 0.08 & 0.07 & 0.340 & $1.14(0.83-1.58)$ & 0.425 \\
\hline rs12642845 & 4 & C/G & 0.21 & 0.24 & 0.272 & $0.87(0.71-1.07)$ & 0.188 \\
\hline
\end{tabular}


Table 3

The association between six SNPs within the TLR6gene and the risk of TB.

\begin{tabular}{|c|c|c|c|c|c|c|}
\hline SNP & Model & Genotype & Cases & Controls & OR $(95 \% \mathrm{Cl})$ & $\mathrm{p}^{\mathrm{a}}$ \\
\hline \multirow[t]{8}{*}{ rs3775073 } & Co-dominant & $\mathrm{G} / \mathrm{G}$ & 245 & 219 & 1.00 & \\
\hline & & $\mathrm{G} / \mathrm{A}$ & 216 & 240 & $0.80(0.62-1.04)$ & 0.090 \\
\hline & & $\mathrm{A} / \mathrm{A}$ & 48 & 49 & $0.88(0.57-1.36)$ & 0.556 \\
\hline & Dominant & $\mathrm{G} / \mathrm{G}$ & 245 & 219 & 1.00 & \\
\hline & & $G / A-A / A$ & 264 & 289 & $0.81(0.63-1.04)$ & 0.100 \\
\hline & Recessive & $G / G-G / A$ & 461 & 459 & 1.00 & \\
\hline & & $\mathrm{A} / \mathrm{A}$ & 48 & 49 & $0.98(0.64-1.49)$ & 0.919 \\
\hline & Log-additive & - & - & - & $0.88(0.73-1.07)$ & 0.191 \\
\hline \multirow[t]{8}{*}{ rs3796508 } & Co-dominant & $\mathrm{G} / \mathrm{G}$ & 471 & 457 & 1.00 & \\
\hline & & $\mathrm{G} / \mathrm{A}$ & 39 & 49 & $0.78(0.50-1.21)$ & 0.262 \\
\hline & & $\mathrm{A} / \mathrm{A}$ & 0 & 2 & - & - \\
\hline & Dominant & $\mathrm{G} / \mathrm{G}$ & 471 & 457 & 1.00 & \\
\hline & & $G / A-A / A$ & 39 & 51 & $0.75(0.48-1.16)$ & 0.190 \\
\hline & Recessive & $G / G-G / A$ & 510 & 506 & 1.00 & \\
\hline & & $\mathrm{A} / \mathrm{A}$ & 0 & 2 & - & - \\
\hline & Log-additive & - & - & - & $0.73(0.48-1.11)$ & 0.139 \\
\hline \multirow[t]{8}{*}{ rs6531668 } & Co-dominant & $\mathrm{G} / \mathrm{G}$ & 294 & 326 & 1.00 & \\
\hline & & $\mathrm{G} / \mathrm{A}$ & 175 & 157 & $1.24(0.95-1.62)$ & 0.118 \\
\hline & & $\mathrm{A} / \mathrm{A}$ & 37 & 25 & $1.65(0.97-2.81)$ & 0.065 \\
\hline & Dominant & $\mathrm{G} / \mathrm{G}$ & 294 & 326 & 1.00 & \\
\hline & & $G / A-A / A$ & 212 & 182 & $1.30(1.01-1.67)$ & $0.046^{*}$ \\
\hline & Recessive & $G / G-G / A$ & 469 & 483 & 1.00 & \\
\hline & & $\mathrm{A} / \mathrm{A}$ & 37 & 25 & $1.53(0.91-2.59)$ & 0.110 \\
\hline & Log-additive & - & - & - & $1.26(1.03-1.55)$ & $0.026^{*}$ \\
\hline \multirow[t]{2}{*}{ rs12644563 } & Co-dominant & $\mathrm{T} / \mathrm{T}$ & 471 & 458 & 1.00 & \\
\hline & & $\mathrm{T} / \mathrm{C}$ & 39 & 47 & $0.81(0.52-1.27)$ & 0.363 \\
\hline
\end{tabular}




\begin{tabular}{|c|c|c|c|c|c|c|}
\hline SNP & Model & Genotype & Cases & Controls & OR $(95 \% \mathrm{Cl})$ & $p^{a}$ \\
\hline & & $\mathrm{C} / \mathrm{C}$ & 0 & 3 & - & - \\
\hline & Dominant & $\mathrm{T} / \mathrm{T}$ & 471 & 458 & 1.00 & \\
\hline & & $\mathrm{T} / \mathrm{C}-\mathrm{C} / \mathrm{C}$ & 39 & 50 & $0.76(0.49-1.18)$ & 0.228 \\
\hline & Recessive & $\mathrm{T} / \mathrm{T}-\mathrm{T} / \mathrm{C}$ & 510 & 505 & 1.00 & \\
\hline & & $\mathrm{C} / \mathrm{C}$ & 0 & 3 & - & - \\
\hline & Log-additive & - & - & - & $0.73(0.48-1.11)$ & 0.144 \\
\hline \multirow[t]{8}{*}{ rs12640631 } & Co-dominant & $\mathrm{T} / \mathrm{T}$ & 430 & 437 & 1.00 & \\
\hline & & $\mathrm{T} / \mathrm{C}$ & 75 & 67 & $1.15(0.80-1.64)$ & 0.453 \\
\hline & & $\mathrm{C} / \mathrm{C}$ & 5 & 4 & $1.26(0.34-4.73)$ & 0.733 \\
\hline & Dominant & $\mathrm{T} / \mathrm{T}$ & 430 & 437 & 1.00 & \\
\hline & & $\mathrm{T} / \mathrm{C}-\mathrm{C} / \mathrm{C}$ & 80 & 71 & $1.15(0.81-1.63)$ & 0.423 \\
\hline & Recessive & $\mathrm{T} / \mathrm{T}-\mathrm{T} / \mathrm{C}$ & 505 & 504 & 1.00 & \\
\hline & & $\mathrm{C} / \mathrm{C}$ & 5 & 4 & $1.24(0.33-4.63)$ & 0.753 \\
\hline & Log-additive & - & - & - & $1.14(0.83-1.57)$ & 0.417 \\
\hline \multirow[t]{8}{*}{ rs12642845 } & Co-dominant & $\mathrm{G} / \mathrm{G}$ & 318 & 289 & 1.00 & \\
\hline & & $\mathrm{G} / \mathrm{C}$ & 165 & 195 & $1.03(0.58-1.82)$ & 0.925 \\
\hline & & $\mathrm{C} / \mathrm{C}$ & 27 & 24 & $0.77(0.59-1.00)$ & 0.050 \\
\hline & Dominant & $\mathrm{G} / \mathrm{G}$ & 318 & 289 & 1.00 & \\
\hline & & $\mathrm{G} / \mathrm{C}-\mathrm{CC}$ & 192 & 219 & $0.80(0.62-1.03)$ & 0.078 \\
\hline & Recessive & $G / G-G / C$ & 483 & 484 & 1.00 & \\
\hline & & $\mathrm{C} / \mathrm{C}$ & 27 & 24 & $1.13(0.64-1.99)$ & 0.665 \\
\hline & Log-additive & - & - & - & $0.87(0.71-1.07)$ & 0.192 \\
\hline
\end{tabular}


Table 4

Age stratification analysis of association between TLR6 and the risk of TB.

\begin{tabular}{|c|c|c|c|c|c|c|}
\hline \multirow[t]{2}{*}{ SNP } & \multirow[t]{2}{*}{ Model } & \multirow[t]{2}{*}{ Genotype } & \multicolumn{2}{|l|}{$\leq 41$} & \multicolumn{2}{|l|}{$>41$} \\
\hline & & & OR $(95 \% \mathrm{Cl})$ & $P^{a}$ & OR (95\% Cl) & $\mathbf{P a}^{\mathbf{a}}$ \\
\hline \multirow[t]{8}{*}{ rs3775073 } & $\begin{array}{l}\text { Co- } \\
\text { dominant }\end{array}$ & $\mathrm{G} / \mathrm{G}$ & 1.00 & & 1.00 & \\
\hline & & $\mathrm{G} / \mathrm{A}$ & $\begin{array}{l}0.76(0.52- \\
1.10)\end{array}$ & 0.141 & $\begin{array}{l}0.80(0.54- \\
1.19)\end{array}$ & 0.275 \\
\hline & & $\mathrm{A} / \mathrm{A}$ & $\begin{array}{l}0.82(0.43- \\
1.57)\end{array}$ & 0.547 & $\begin{array}{l}0.86(0.45- \\
1.63)\end{array}$ & 0.641 \\
\hline & Dominant & $G / G$ & 1.00 & & 1.00 & \\
\hline & & $\mathrm{G} / \mathrm{A}-\mathrm{A} / \mathrm{A}$ & $\begin{array}{l}0.77(0.54- \\
1.09)\end{array}$ & 0.143 & $\begin{array}{l}0.81(0.56- \\
1.18)\end{array}$ & 0.279 \\
\hline & Recessive & $\begin{array}{l}\mathrm{G} / \mathrm{G}- \\
\mathrm{G} / \mathrm{A}\end{array}$ & 1.00 & & 1.00 & \\
\hline & & $\mathrm{A} / \mathrm{A}$ & $\begin{array}{l}0.94(0.51- \\
1.76)\end{array}$ & 0.855 & $\begin{array}{l}0.95(0.52- \\
1.76)\end{array}$ & 0.880 \\
\hline & Log-additive & - & $\begin{array}{l}0.84(0.64- \\
1.11)\end{array}$ & 0.221 & $\begin{array}{l}0.88(0.66- \\
1.17)\end{array}$ & 0.377 \\
\hline \multirow[t]{8}{*}{ rs3796508 } & $\begin{array}{l}\text { Co- } \\
\text { dominant }\end{array}$ & $\mathrm{G} / \mathrm{G}$ & 1.00 & & 1.00 & \\
\hline & & $\mathrm{G} / \mathrm{A}$ & $\begin{array}{l}0.95(0.49- \\
1.85)\end{array}$ & 0.884 & - & - \\
\hline & & $\mathrm{A} / \mathrm{A}$ & - & - & - & - \\
\hline & Dominant & $\mathrm{G} / \mathrm{G}$ & 1.00 & & 1.00 & \\
\hline & & $G / A-A / A$ & $\begin{array}{l}0.92(0.48- \\
1.76)\end{array}$ & 0.792 & $\begin{array}{l}0.60(0.32- \\
1.14)\end{array}$ & 0.118 \\
\hline & Recessive & $\begin{array}{l}\mathrm{G} / \mathrm{G}- \\
\mathrm{G} / \mathrm{A}\end{array}$ & 1.00 & & 1.00 & \\
\hline & & $\mathrm{A} / \mathrm{A}$ & - & - & - & - \\
\hline & Log-additive & - & $\begin{array}{l}0.89(0.47- \\
1.66)\end{array}$ & 0.706 & $\begin{array}{l}0.60(0.32- \\
1.14)\end{array}$ & 0.118 \\
\hline \multirow[t]{2}{*}{ rs6531668 } & $\begin{array}{l}\text { Co- } \\
\text { dominant }\end{array}$ & $G / G$ & 1.00 & & 1.00 & \\
\hline & & $\mathrm{G} / \mathrm{A}$ & $\begin{array}{l}1.10(0.76- \\
1.61)\end{array}$ & 0.613 & $\begin{array}{l}1.75(1.14- \\
2.68)\end{array}$ & $0.010^{*}$ \\
\hline
\end{tabular}




\begin{tabular}{|c|c|c|c|c|c|c|}
\hline \multirow[t]{8}{*}{ SNP } & \multirow[t]{3}{*}{ Model } & \multirow[t]{2}{*}{ Genotype } & \multicolumn{2}{|l|}{$\leq 41$} & \multicolumn{2}{|l|}{$>41$} \\
\hline & & & OR (95\% Cl) & $P^{a}$ & OR $(95 \% \mathrm{Cl})$ & $\mathbf{P a}^{\mathbf{a}}$ \\
\hline & & $\mathrm{A} / \mathrm{A}$ & $\begin{array}{l}1.73(0.76- \\
3.95)\end{array}$ & 0.194 & $\begin{array}{l}1.83(0.87- \\
3.85)\end{array}$ & 0.111 \\
\hline & Dominant & $\mathrm{G} / \mathrm{G}$ & 1.00 & & 1.00 & \\
\hline & & $\mathrm{G} / \mathrm{A}-\mathrm{A} / \mathrm{A}$ & $\begin{array}{l}1.17(0.81- \\
1.67)\end{array}$ & 0.405 & $\begin{array}{l}1.77(1.19- \\
2.62)\end{array}$ & $0.005^{*}$ \\
\hline & Recessive & $\begin{array}{l}\mathrm{G} / \mathrm{G}- \\
\mathrm{G} / \mathrm{A}\end{array}$ & 1.00 & & 1.00 & \\
\hline & & $\mathrm{A} / \mathrm{A}$ & $\begin{array}{l}1.67(0.74- \\
3.76)\end{array}$ & 0.219 & $\begin{array}{l}1.53(0.74- \\
3.17)\end{array}$ & 0.256 \\
\hline & Log-additive & - & $\begin{array}{l}1.19(0.88- \\
1.61)\end{array}$ & 0.250 & $\begin{array}{l}1.51(1.11- \\
2.06)\end{array}$ & $0.009^{*}$ \\
\hline \multirow[t]{8}{*}{ rs12644563 } & $\begin{array}{l}\text { Co- } \\
\text { dominant }\end{array}$ & $\mathrm{T} / \mathrm{T}$ & 1.00 & & 1.00 & \\
\hline & & $\mathrm{T} / \mathrm{C}$ & $\begin{array}{l}1.00(0.51- \\
1.97)\end{array}$ & 0.998 & - & - \\
\hline & & $\mathrm{C} / \mathrm{C}$ & - & - & - & - \\
\hline & Dominant & $\mathrm{T} / \mathrm{T}$ & 1.00 & & 1.00 & \\
\hline & & $\mathrm{T} / \mathrm{C}-\mathrm{C} / \mathrm{C}$ & $\begin{array}{l}0.89(0.46- \\
1.73)\end{array}$ & 0.736 & $\begin{array}{l}0.67(0.36- \\
1.26)\end{array}$ & 0.212 \\
\hline & Recessive & $\mathrm{T} / \mathrm{T}-\mathrm{T} / \mathrm{C}$ & 1.00 & & 1.00 & \\
\hline & & $\mathrm{C} / \mathrm{C}$ & - & - & - & - \\
\hline & Log-additive & - & $\begin{array}{l}0.82(0.44- \\
1.51)\end{array}$ & 0.517 & $\begin{array}{l}0.67(0.36- \\
1.26)\end{array}$ & 0.212 \\
\hline \multirow[t]{6}{*}{ rs12640631 } & $\begin{array}{l}\text { Co- } \\
\text { dominant }\end{array}$ & $\mathrm{T} / \mathrm{T}$ & 1.00 & & 1.00 & \\
\hline & & $\mathrm{T} / \mathrm{C}$ & $\begin{array}{l}1.03(0.61- \\
1.74)\end{array}$ & 0.913 & $\begin{array}{l}1.23(0.73- \\
2.07)\end{array}$ & 0.437 \\
\hline & & $\mathrm{C} / \mathrm{C}$ & $\begin{array}{l}0.73(0.13- \\
4.08)\end{array}$ & 0.719 & - & - \\
\hline & Dominant & $\mathrm{T} / \mathrm{T}$ & 1.00 & & 1.00 & \\
\hline & & $\mathrm{T} / \mathrm{C}-\mathrm{C} / \mathrm{C}$ & $\begin{array}{l}1.00(0.60- \\
1.67)\end{array}$ & 0.991 & $\begin{array}{l}1.29(0.77- \\
2.16)\end{array}$ & 0.335 \\
\hline & Recessive & $\mathrm{T} / \mathrm{T}-\mathrm{T} / \mathrm{C}$ & 1.00 & & 1.00 & \\
\hline
\end{tabular}




\begin{tabular}{|c|c|c|c|c|c|c|}
\hline \multirow[t]{2}{*}{ SNP } & \multirow[t]{2}{*}{ Model } & \multirow[t]{2}{*}{ Genotype } & \multicolumn{2}{|l|}{$\leq 41$} & \multicolumn{2}{|l|}{$>41$} \\
\hline & & & OR (95\% Cl) & $\mathrm{P}^{\mathrm{a}}$ & OR $(95 \% \mathrm{Cl})$ & $\mathrm{P}^{\mathrm{a}}$ \\
\hline & & $\mathrm{C} / \mathrm{C}$ & $\begin{array}{l}0.73(0.13- \\
4.06)\end{array}$ & 0.716 & - & - \\
\hline & Log-additive & - & $\begin{array}{l}0.98(0.62- \\
1.55)\end{array}$ & 0.931 & $1.34(0.81-2.2)$ & 0.255 \\
\hline \multirow[t]{8}{*}{ rs12642845 } & $\begin{array}{l}\text { Co- } \\
\text { dominant }\end{array}$ & $G / G$ & 1.00 & & 1.00 & \\
\hline & & $\mathrm{G} / \mathrm{C}$ & $\begin{array}{l}0.82(0.56- \\
1.19)\end{array}$ & 0.293 & $\begin{array}{l}0.62(0.42- \\
0.93)\end{array}$ & $0.021^{*}$ \\
\hline & & $\mathrm{C} / \mathrm{C}$ & $\begin{array}{l}0.92(0.40- \\
2.12)\end{array}$ & 0.847 & $\begin{array}{l}1.09(0.46- \\
2.56)\end{array}$ & 0.845 \\
\hline & Dominant & $\mathrm{G} / \mathrm{G}$ & 1.00 & & 1.00 & \\
\hline & & $\mathrm{G} / \mathrm{C}-\mathrm{CC}$ & $\begin{array}{l}0.83(0.58- \\
1.19)\end{array}$ & 0.310 & $\begin{array}{l}0.67(0.46- \\
0.99)\end{array}$ & $0.042^{*}$ \\
\hline & Recessive & $\mathrm{G} / \mathrm{G}-\mathrm{G} / \mathrm{C}$ & 1.00 & & 1.00 & \\
\hline & & $\mathrm{C} / \mathrm{C}$ & $\begin{array}{l}1.00(0.44- \\
2.27)\end{array}$ & 0.992 & $\begin{array}{l}1.30(0.56- \\
3.01)\end{array}$ & 0.541 \\
\hline & Log-additive & - & $\begin{array}{l}0.88(0.65- \\
1.19)\end{array}$ & 0.392 & $\begin{array}{l}0.79(0.58- \\
1.09)\end{array}$ & 0.152 \\
\hline
\end{tabular}


Table 5

Gender stratification analysis of association between TLR6 and the risk of TB.

\begin{tabular}{|c|c|c|c|c|c|c|}
\hline \multirow[t]{2}{*}{ SNP } & \multirow[t]{2}{*}{ Model } & \multirow[t]{2}{*}{ Genotype } & \multicolumn{2}{|l|}{ Males } & \multicolumn{2}{|l|}{ Females } \\
\hline & & & OR (95\% Cl) & $P^{a}$ & OR (95\% Cl) & $P^{a}$ \\
\hline \multirow[t]{8}{*}{ rs3775073 } & $\begin{array}{l}\text { Co- } \\
\text { dominant }\end{array}$ & $G / G$ & 1.00 & & 1.00 & \\
\hline & & $\mathrm{G} / \mathrm{A}$ & $\begin{array}{l}0.70(0.50- \\
0.98)\end{array}$ & $0.036^{*}$ & $\begin{array}{l}0.99(0.66- \\
1.51)\end{array}$ & 0.980 \\
\hline & & $\mathrm{A} / \mathrm{A}$ & $\begin{array}{l}0.91(0.54- \\
1.53)\end{array}$ & 0.712 & $\begin{array}{l}0.80(0.35- \\
1.81)\end{array}$ & 0.589 \\
\hline & Dominant & $\mathrm{G} / \mathrm{G}$ & 1.00 & & 1.00 & \\
\hline & & $G / A-A / A$ & $\begin{array}{l}0.74(0.54- \\
1.01)\end{array}$ & 0.059 & $\begin{array}{l}0.97(0.65- \\
1.45)\end{array}$ & 0.872 \\
\hline & Recessive & $\begin{array}{l}\mathrm{G} / \mathrm{G}- \\
\mathrm{G} / \mathrm{A}\end{array}$ & 1.00 & & 1.00 & \\
\hline & & $\mathrm{A} / \mathrm{A}$ & $\begin{array}{l}1.08(0.65- \\
1.77)\end{array}$ & 0.776 & $\begin{array}{l}0.80(0.36- \\
1.76)\end{array}$ & 0.579 \\
\hline & Log-additive & - & $\begin{array}{l}0.86(0.68- \\
1.09)\end{array}$ & 0.202 & $\begin{array}{l}0.94(0.68- \\
1.31)\end{array}$ & 0.716 \\
\hline \multirow[t]{8}{*}{ rs3796508 } & $\begin{array}{l}\text { Co- } \\
\text { dominant }\end{array}$ & $G / G$ & 1.00 & & 1.00 & \\
\hline & & $\mathrm{G} / \mathrm{A}$ & $0.86(0.5-1.49)$ & 0.594 & $\begin{array}{l}0.66(0.32- \\
1.38)\end{array}$ & 0.272 \\
\hline & & $\mathrm{A} / \mathrm{A}$ & - & - & - & - \\
\hline & Dominant & $\mathrm{G} / \mathrm{G}$ & 1.00 & & 1.00 & \\
\hline & & $\mathrm{G} / \mathrm{A}-\mathrm{A} / \mathrm{A}$ & $\begin{array}{l}0.83(0.48- \\
1.44)\end{array}$ & 0.511 & $\begin{array}{l}0.63(0.30- \\
1.30)\end{array}$ & 0.213 \\
\hline & Recessive & $\begin{array}{l}\mathrm{G} / \mathrm{G}- \\
\mathrm{G} / \mathrm{A}\end{array}$ & 1.00 & & 1.00 & \\
\hline & & $\mathrm{A} / \mathrm{A}$ & - & - & - & - \\
\hline & Log-additive & - & $\begin{array}{l}0.81(0.47- \\
1.38)\end{array}$ & 0.438 & $\begin{array}{l}0.61(0.30- \\
1.24)\end{array}$ & 0.174 \\
\hline \multirow[t]{2}{*}{ rs6531668 } & $\begin{array}{l}\text { Co- } \\
\text { dominant }\end{array}$ & $G / G$ & 1.00 & & 1.00 & \\
\hline & & $\mathrm{G} / \mathrm{A}$ & $\begin{array}{l}1.12(0.80- \\
1.58)\end{array}$ & 0.507 & $\begin{array}{l}1.41(0.92- \\
2.18)\end{array}$ & 0.116 \\
\hline
\end{tabular}




\begin{tabular}{|c|c|c|c|c|c|c|}
\hline \multirow[t]{8}{*}{ SNP } & \multirow[t]{2}{*}{ Model } & \multirow[t]{2}{*}{ Genotype } & \multicolumn{2}{|l|}{ Males } & \multicolumn{2}{|l|}{ Females } \\
\hline & & & OR (95\% Cl) & $P^{a}$ & OR (95\% Cl) & $P^{a}$ \\
\hline & & $\mathrm{A} / \mathrm{A}$ & $\begin{array}{l}1.57(0.82- \\
3.00)\end{array}$ & 0.173 & $\begin{array}{l}1.76(0.69- \\
4.48)\end{array}$ & 0.237 \\
\hline & Dominant & $\mathrm{G} / \mathrm{G}$ & 1.00 & & 1.00 & \\
\hline & & $\mathrm{G} / \mathrm{A}-\mathrm{A} / \mathrm{A}$ & $\begin{array}{l}1.19(0.86- \\
1.64)\end{array}$ & 0.291 & $\begin{array}{l}1.45(0.96- \\
2.20)\end{array}$ & 0.077 \\
\hline & Recessive & $\begin{array}{l}\mathrm{G} / \mathrm{G}- \\
\mathrm{G} / \mathrm{A}\end{array}$ & 1.00 & & 1.00 & \\
\hline & & $\mathrm{A} / \mathrm{A}$ & $\begin{array}{l}1.51(0.80- \\
2.85)\end{array}$ & 0.206 & $\begin{array}{l}1.54(0.61- \\
3.88)\end{array}$ & 0.357 \\
\hline & Log-additive & - & $\begin{array}{l}1.19(0.92- \\
1.54)\end{array}$ & 0.179 & $\begin{array}{l}1.37(0.97- \\
1.94)\end{array}$ & 0.071 \\
\hline \multirow[t]{8}{*}{ rs12644563 } & $\begin{array}{l}\text { Co- } \\
\text { dominant }\end{array}$ & $\mathrm{T} / \mathrm{T}$ & 1.00 & & 1.00 & \\
\hline & & $\mathrm{T} / \mathrm{C}$ & $\begin{array}{l}0.89(0.51- \\
1.56)\end{array}$ & 0.693 & $\begin{array}{l}0.70(0.33- \\
1.48)\end{array}$ & 0.351 \\
\hline & & $\mathrm{C} / \mathrm{C}$ & - & - & - & - \\
\hline & Dominant & $\mathrm{T} / \mathrm{T}$ & 1.00 & & 1.00 & \\
\hline & & $\mathrm{T} / \mathrm{C}-\mathrm{C} / \mathrm{C}$ & $\begin{array}{l}0.83(0.48- \\
1.44)\end{array}$ & 0.513 & $\begin{array}{l}0.67(0.32- \\
1.39)\end{array}$ & 0.278 \\
\hline & Recessive & $\mathrm{T} / \mathrm{T}-\mathrm{T} / \mathrm{C}$ & 1.00 & & 1.00 & \\
\hline & & $\mathrm{C} / \mathrm{C}$ & - & - & - & - \\
\hline & Log-additive & - & $\begin{array}{l}0.79(0.47- \\
1.33)\end{array}$ & 0.373 & $\begin{array}{l}0.65(0.32- \\
1.31)\end{array}$ & 0.227 \\
\hline \multirow[t]{6}{*}{ rs12640631 } & $\begin{array}{l}\text { Co- } \\
\text { dominant }\end{array}$ & $\mathrm{T} / \mathrm{T}$ & 1.00 & & 1.00 & \\
\hline & & $\mathrm{T} / \mathrm{C}$ & $\begin{array}{l}1.18(0.75- \\
1.85)\end{array}$ & 0.479 & $\begin{array}{l}1.11(0.62- \\
1.98)\end{array}$ & 0.722 \\
\hline & & $\mathrm{C} / \mathrm{C}$ & $\begin{array}{l}0.62(0.10- \\
3.78)\end{array}$ & 0.607 & $\begin{array}{l}3.06(0.31- \\
2.73)\end{array}$ & 0.336 \\
\hline & Dominant & $\mathrm{T} / \mathrm{T}$ & 1.00 & & 1.00 & \\
\hline & & $\mathrm{T} / \mathrm{C}-\mathrm{C} / \mathrm{C}$ & $\begin{array}{l}1.14(0.73- \\
1.77)\end{array}$ & 0.566 & $\begin{array}{l}1.18(0.68- \\
2.07)\end{array}$ & 0.557 \\
\hline & Recessive & $\mathrm{T} / \mathrm{T}-\mathrm{T} / \mathrm{C}$ & 1.00 & & 1.00 & \\
\hline
\end{tabular}




\begin{tabular}{|c|c|c|c|c|c|c|}
\hline \multirow[t]{4}{*}{ SNP } & \multirow[t]{2}{*}{ Model } & \multirow[t]{2}{*}{ Genotype } & \multicolumn{2}{|l|}{ Males } & \multicolumn{2}{|l|}{ Females } \\
\hline & & & OR $(95 \% \mathrm{Cl})$ & $\mathrm{P}^{\mathrm{a}}$ & OR $(95 \% \mathrm{Cl})$ & $P^{a}$ \\
\hline & & $\mathrm{C} / \mathrm{C}$ & $\begin{array}{l}0.61(0.10- \\
3.70)\end{array}$ & 0.591 & $\begin{array}{l}3.01(0.31- \\
2.25)\end{array}$ & 0.342 \\
\hline & Log-additive & - & $\begin{array}{l}1.09(0.72- \\
1.64)\end{array}$ & 0.686 & $\begin{array}{l}1.23(0.74- \\
2.04)\end{array}$ & 0.431 \\
\hline \multirow[t]{8}{*}{ rs12642845 } & $\begin{array}{l}\text { Co- } \\
\text { dominant }\end{array}$ & $G / G$ & 1.00 & & 1.00 & \\
\hline & & $\mathrm{G} / \mathrm{C}$ & $\begin{array}{l}0.71(0.51- \\
0.99)\end{array}$ & $0.042^{*}$ & $\begin{array}{l}0.90(0.59- \\
1.38)\end{array}$ & 0.627 \\
\hline & & $\mathrm{C} / \mathrm{C}$ & $\begin{array}{l}1.34(0.64- \\
2.79)\end{array}$ & 0.439 & $\begin{array}{l}0.70(0.27- \\
1.80)\end{array}$ & 0.455 \\
\hline & Dominant & $G / G$ & 1.00 & & 1.00 & \\
\hline & & $\mathrm{G} / \mathrm{C}-\mathrm{CC}$ & $\begin{array}{l}0.77(0.56- \\
1.05)\end{array}$ & 0.102 & $\begin{array}{l}0.87(0.58- \\
1.32)\end{array}$ & 0.514 \\
\hline & Recessive & $G / G-G / C$ & 1.00 & & 1.00 & \\
\hline & & $\mathrm{C} / \mathrm{C}$ & $\begin{array}{l}1.52(0.74- \\
3.14)\end{array}$ & 0.257 & $\begin{array}{l}0.73(0.28- \\
1.85)\end{array}$ & 0.501 \\
\hline & Log-additive & - & $\begin{array}{l}0.88(0.68- \\
1.15)\end{array}$ & 0.350 & $\begin{array}{l}0.87(0.62- \\
1.23)\end{array}$ & 0.428 \\
\hline
\end{tabular}


Table 6

Haplotype analysis of TLR6 SNPs in association with the risk of

TB.

\begin{tabular}{|lllll|}
\hline Haplotype & Freq & & & \\
\cline { 2 - 5 } & Cases & Controls & OR $(95 \% \mathrm{Cl})$ & $\mathbf{P}^{\mathrm{a}}$ \\
\hline TTA & 0.87 & 0.88 & $0.87(0.66-1.13)$ & 0.284 \\
\hline CTA & 0.74 & 0.91 & $0.74(0.55-0.99)$ & $0.043^{*}$ \\
\hline CCG & 0.90 & 0.33 & $0.90(0.74-1.09)$ & 0.282 \\
\hline CTG & 1.08 & 0.54 & $1.08(0.90-1.28)$ & 0.418 \\
\hline TTC & 0.87 & 0.24 & $0.87(0.71-1.07)$ & 0.192 \\
\hline CCG & 0.73 & 0.05 & $0.73(0.48-1.11)$ & 0.144 \\
\hline TCG & 0.48 & 0.98 & $0.47(0.28-0.79)$ & $0.004^{*}$ \\
\hline TTG & 0.93 & 0.31 & $0.93(0.77-1.13)$ & 0.479 \\
\hline
\end{tabular}

\section{Discussion}

Numerous evidences suggested that host genetic variants confer enhanced TB risk, and several candidate genes have been demonstrated, such as the CYP7A1 gene, Vitamin D receptor gene, the CD14 gene and the TLRs family genes [16-19]. Nevertheless, the relationship between TLR6 polymorphism and TB risk in Chinese population is poorly understand. In the present study, TLR6 polymorphisms and TB susceptibility was investigated. Our results revealed that rs6531668 was significantly correlated with an increased the risk of TB. Next, stratified analyses discovered significant effect of rs6531668 and rs 12642845 of $T L R 6$ on the risk of TB in age $>41$ years, and rs $3775073 \mathrm{G} / \mathrm{A}$ genotype and rs 12642845 $\mathrm{G} / \mathrm{C}$ genotype were decreased the TB risk in males.

During the past studies reported that the innate immune system can enables to recognize invading bacterial such as Mtb, and have a crucial role in host defense mechanism. In human, TLRs are thought to recognition of pathogens and influence the pathogenesis of the immune response [8, 20,21]. Studies have been provided evidences that LTRs can recognize PAMPs of Mtb and initiate our innate immune response, ultimately culminating in antimicrobial host defense $[22,23]$. Hence, the TLRs family genes have become biologically plausible candidate genes in studies of TB risk [24].

$T L R 6$ is $2.3 \mathrm{~kb}$ in length and located on chromosome 4. TLR6 encodes a 796-amino acid type I transmembrane protein with a 21 aa transmembrane domain, a 630aa extracellular leucine-rich repeat region and a 145aa intra-cellular Toll/IL-1R signaling domain [25]. TLR6 belongs to TLR2 subfamily, and forms heterodimers with TLR2, resulting in the recognition of Mtb [19]. Multiple studies confirmed that 
$T L R 6$ gene polymorphisms have been associated with TB risk [22, 23, 25]. In the study of Musse et al. due to $T L R 6$ variants were deficient in ability to mediate signal transduction, they found significant association between TLR6 polymorphisms and increased TB sensibility in African Americans [26]. Shey et al. suggested that $T L R \sigma$ variants reduced NF-kB signaling which led to an altered level of IL-6 production and thus protection against TB disease [25]. While Randhawa et al. reported that TLR6 variants leads to increased IFN-y production and thus resistance to TB disease [22]. Xu et al. was not confirmed that TLR6 variants was related with the risk of TB in Chinese population [23]. However, our results demonstrated that $T L R 6$ variants (rs6531668, rs3775073, rs12642845) were significantly related with TB risk.

Furthermore, Regulome DB and Haploreg v4.1 suggested that rs6531688 and rs3775073 were the binding site for the transcription factor. Thus, further characterization is taken into account to investigate in vivo and in vitro at the molecular level.

In summary, our results confirmed that rs6531668, rs 3775073 and rs 12642845 of $T L R 6$ were significantly correlated with TB risk in Chinese population. Our work provided an evidence to estimate the relationship between TLR6 polymorphism and TB susceptibility.

\section{Abbreviations}

Cls

confidence intervals

EDTA

ethylenediaminetetraacetic acid

GWAS

genome-wide association studies

HWE

Hardy-Weinberg equilibrium

LD

Linkage disequilibrium

MAF

minor allele frequency

ORs

odds ratios

PAMPs

pathogen-associated molecular patterns

TB

Tuberculosis

TLR

toll-like receptor 


\section{Declarations}

\section{Authors' contributions}

GC designed the research; DC wrote the paper; WL analyzed the data; LQ collected the data; $L Y, D L$ and GZ performed research. All authors have read and. agreed with the final manuscript.

\section{Funding}

This study was supported by the Key Research and Development Program of Shaanxi (No. 2018SF-222).

\section{Availability of data and materials}

The datasets used and/or analyzed during the current study are available from the corresponding author on reasonable request.

\section{Ethics approval and consent to participate}

The study approved by the Ethical Committee of the Xi'an Chest Hospital, and the informed consent documents were signed by all participants.

\section{Consent for publication}

Not applicable

\section{Competing interests}

The authors declare that they have no competing interests.

Acknowledgments

We are grateful to all participants for providing blood samples. We also appreciate the reviewers and editors for their efforts and patience.

\section{References}

1. Casanova JL, Abel L, Quintana-Murci L. Human TLRs and IL-1Rs in host defense: natural insights from evolutionary, epidemiological, and clinical genetics. Annu Rev Immunol. 2011;29:447-91.

2. Chen C, Liu Q, Zhu L, Yang H, Lu W. Vitamin D receptor gene polymorphisms on the risk of tuberculosis, a meta-analysis of 29 case-control studies. PloS one. 2013;8(12):e83843.

3. Ladefoged K, Rendal T, Skifte T, Andersson M, Soborg B, Koch A. Risk factors for tuberculosis in Greenland: case-control study. The international journal of tuberculosis lung disease: the official journal of the International Union against Tuberculosis Lung Disease. 2011;15(1):44-9. 
4. Murray M, Oxlade $\mathrm{O}$, Lin $\mathrm{HH}$. Modeling social, environmental and biological determinants of tuberculosis. The international journal of tuberculosis lung disease: the official journal of the International Union against Tuberculosis Lung Disease. 2011;15(Suppl 2):64-70.

5. Du B, Hua D, Droma C, Zhang H, Qu J, Yang L, Jin T, Yang J. Association between TAP2 and SEC14L2 polymorphisms and pulmonary tuberculosis risk in the Tibetan Chinese population. INTERNATIONAL JOURNAL OF CLINICAL AND EXPERIMENTAL PATHOLOGY. 2017;10(11):11188-94.

6. Hu Q, Zhang M, He N, Liang G, Xu S, Wang Y, Mo F, Zhang H, Kang L, Jin T. Association of genetic polymorphisms with pulmonary tuberculosis in a Chinese Tibetan population: a case-control study. Int J Clin Exp Pathol. 2016;9(1):267-74.

7. Castrillon DM, Mejia SA, Hernandez JM, Robledo J. Pulmonary tuberculosis in a 4-month-old twin: hereditary versus environmental factors. The international journal of tuberculosis lung disease: the official journal of the International Union against Tuberculosis Lung Disease. 2013;17(6):848-9.

8. Misch EA, Hawn TR. Toll-like receptor polymorphisms and susceptibility to human disease. Clinical science (London England: 1979). 2008;114(5):347-60.

9. Azad AK, Sadee W, Schlesinger LS. Innate immune gene polymorphisms in tuberculosis. Infect Immun. 2012;80(10):3343-59.

10. Thada S, Valluri VL, Gaddam SL. Influence of Toll-like receptor gene polymorphisms to tuberculosis susceptibility in humans. Scand J Immunol. 2013;78(3):221-9.

11. Velez DR, Wejse C, Stryjewski ME, Abbate E, Hulme WF, Myers JL, Estevan R, Patillo SG, Olesen R, Tacconelli A, et al. Variants in toll-like receptors 2 and 9 influence susceptibility to pulmonary tuberculosis in Caucasians, African-Americans, and West Africans. Human genetics. 2010;127(1):65-73.

12. Ocejo-Vinyals JG, Puente de Mateo E, Ausin F, Aguero R, Arroyo JL, Gutierrez-Cuadra M, Farinas MC. Human toll-like receptor $1 \mathrm{~T} 1805 \mathrm{G}$ polymorphism and susceptibility to pulmonary tuberculosis in northern Spain. The international journal of tuberculosis lung disease: the official journal of the International Union against Tuberculosis Lung Disease. 2013;17(5):652-4.

13. Zaki HY, Leung KH, Yiu WC, Gasmelseed N, Elwali NE, Yip SP. Common polymorphisms in TLR4 gene associated with susceptibility to pulmonary tuberculosis in the Sudanese. The international journal of tuberculosis lung disease: the official journal of the International Union against Tuberculosis Lung Disease. 2012;16(7):934-40.

14. Torres-Garcia D, Cruz-Lagunas A, Garcia-Sancho Figueroa MC, Fernandez-Plata R, Baez-Saldana R, Mendoza-Milla C, Barquera R, Carrera-Eusebio A, Ramirez-Bravo S, Campos L, et al. Variants in tolllike receptor 9 gene influence susceptibility to tuberculosis in a Mexican population. Journal of translational medicine. 2013;11:220.

15. Zhu L, Liu L, He X, Yan M, Du J, Yang H, Zhang Y, Yuan D, Jin T. Association between genetic polymorphism of telomere-associated gene ACYP2 and the risk of HAPE among the Chinese Han population: A Case-control study. Medicine. 2017;96(13):e6504. 
16. Sun L, Hu C, Zheng C, Qian Y, Liang Q, Lv Z, Huang Z, Qi K, Gong H, Zhang Z, et al. F0X03 variants are beneficial for longevity in Southern Chinese living in the Red River Basin: A case-control study and meta-analysis. Scientific reports. 2015;5:9852.

17. Qrafli M, Amar Y, Bourkadi J, Ben Amor J, Iraki G, Bakri Y, Amzazi S, Lahlou O, Seghrouchni F, El Aouad R, et al. The CYP7A1 gene rs3808607 variant is associated with susceptibility of tuberculosis in Moroccan population. The Pan African medical journal. 2014;18:1.

18. Miao R, Ge H, Xu L, Xu F. CD14 -159C/T polymorphism contributes to the susceptibility to tuberculosis: evidence from pooled 1,700 cases and 1,816 controls. Mol Biol Rep. 2014;41(5):34816.

19. Zhang Y, Jiang T, Yang X, Xue Y, Wang C, Liu J, Zhang X, Chen Z, Zhao M, Li JC. Toll-like receptor - 1, -2 , and -6 polymorphisms and pulmonary tuberculosis susceptibility: a systematic review and metaanalysis. PloS one. 2013;8(5):e63357.

20. Fujiwara N, Porcelli SA, Naka T, Yano I, Maeda S, Kuwata H, Akira S, Uematsu S, Takii T, Ogura H, et al. Bacterial sphingophospholipids containing non-hydroxy fatty acid activate murine macrophages via Toll-like receptor 4 and stimulate bacterial clearance. Biochim Biophys Acta. 2013;1831(6):117784.

21. He S, Liang Y, Shao F, Wang X. Toll-like receptors activate programmed necrosis in macrophages through a receptor-interacting kinase-3-mediated pathway. Proc Natl Acad Sci USA. 2011;108(50):20054-9.

22. Randhawa AK, Shey MS, Keyser A, Peixoto B, Wells RD, de Kock M, Lerumo L, Hughes J, Hussey G, Hawkridge A, et al. Association of human TLR1 and TLR6 deficiency with altered immune responses to BCG vaccination in South African infants. PLoS pathogens. 2011;7(8):e1002174.

23. Wu L, Hu Y, Li D, Jiang W, Xu B. Screening toll-like receptor markers to predict latent tuberculosis infection and subsequent tuberculosis disease in a Chinese population. BMC medical genetics. 2015;16:19.

24. Qian C, Cao X. Regulation of Toll-like receptor signaling pathways in innate immune responses. Ann N Y Acad Sci. 2013;1283:67-74.

25. Shey MS, Randhawa AK, Bowmaker M, Smith E, Scriba TJ, de Kock M, Mahomed H, Hussey G, Hawn TR, Hanekom WA. Single nucleotide polymorphisms in toll-like receptor 6 are associated with altered lipopeptide- and mycobacteria-induced interleukin-6 secretion. Genes Immun. 2010;11(7):561-72.

26. Ma X, Liu Y, Gowen BB, Graviss EA, Clark AG, Musser JM. Full-exon resequencing reveals toll-like receptor variants contribute to human susceptibility to tuberculosis disease. PloS one. 2007;2(12):e1318.

\section{Figures}



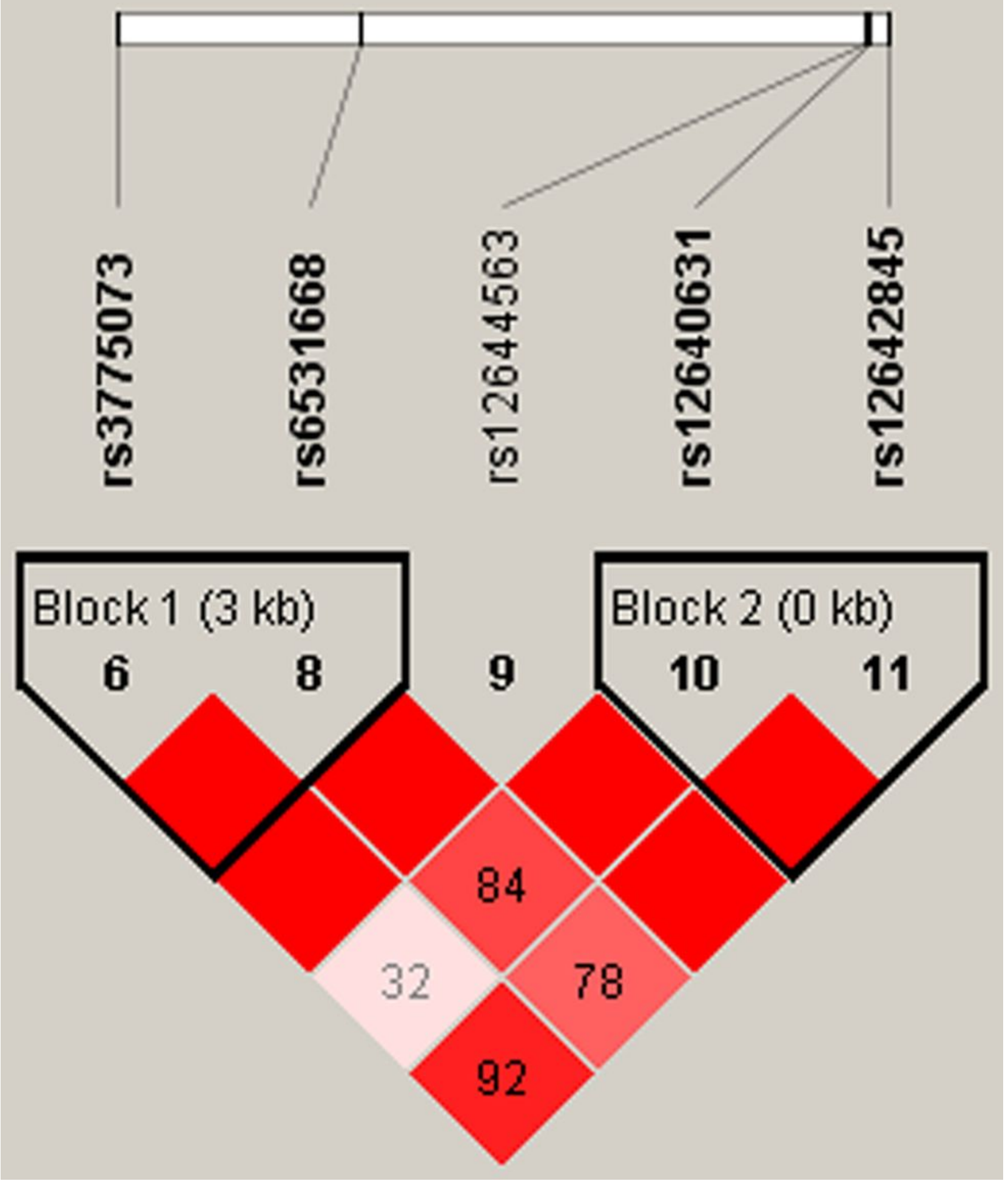

Figure 1

Haplotype block map for SNPs in the TLR6 gene. The LD between each pair of SNPs is standardized D'. The bright red corresponds to a very strong LD; white corresponds to no LD; and pink corresponds to intermediate LD.

\section{Supplementary Files}


This is a list of supplementary files associated with this preprint. Click to download.

- SupplementaryTable1.docx 